\title{
A thermostable oral SARS-CoV-2 vaccine induces mucosal and protective immunity
}

Bertrand Bellier ${ }^{1}$, Alicia Saura ${ }^{2,3}$, Lucas A. Luján ${ }^{2,3}$, Cecilia R. Molina ${ }^{2,3}$, Hugo D. Lujan ${ }^{2,3,{ }^{*} \text {, }}$ and David Klatzmann ${ }^{1,4, *}$

${ }^{1}$ Sorbonne Université, INSERM, UMRS 959, Immunology-Immunopathology-

Immunotherapy, i3, F75013 Paris, France

${ }^{2}$ Centro de Investigación y Desarrollo en Inmunología y Enfermedades Infecciosas (CIDIE), Consejo Nacional de Investigaciones Científicas y Técnicas (CONICET)/Universidad Católica de Córdoba (UCC). Córdoba, Argentina.

${ }^{3}$ Facultad de Ciencias de la Salud, Universidad Católica de Córdoba (UCC). Córdoba, Argentina.

${ }^{4}$ AP-HP, Hôpital Pitié-Salpêtrière, Clinical Investigation Center for Biotherapies (CIC-BTi) and Immunology-Inflammation-Infectiology and Dermatology Department (3iD), Paris, France

*Correspondence and requests for materials should be addressed to Hugo D. Lujan (hlujan@ucc.edu.ar) and David Klatzmann (david.klatzmann@sorbonne-universite.fr) 


\section{Abstract}

2 An ideal protective vaccine against SARS-CoV-2 should not only be effective in preventing

3 disease, but also in preventing virus transmission. It should also be well accepted by the

4 population and have a simple logistic chain. To fulfill these criteria, we developed a

5 thermostable, orally administered vaccine that can induce a robust mucosal neutralizing

6 immune response. We used our platform based on retrovirus-derived enveloped virus-like

7 particles (e-VLPs) harnessed with variable surface proteins (VSPs) from the intestinal

8 parasite Giardia lamblia, affording them resistance to degradation and the triggering of

9 robust mucosal cellular and antibody immune responses after oral administration. We

10 made e-VLPs expressing various forms of the SARS-CoV-2 Spike protein (S), with or

11 without membrane protein (M) expression. We found that prime-boost administration of

12 VSP-decorated e-VLPs expressing a pre-fusion stabilized form of S and M triggers robust

13 mucosal responses against SARS-CoV-2 in mice and hamsters, which translate into

14 complete protection from a viral challenge. Moreover, they dramatically boosted the IgA

15 mucosal response of intramuscularly injected vaccines. We conclude that our

16 thermostable orally administered e-VLP vaccine could be a valuable addition to the

17 current arsenal against SARS-CoV-2, in a stand-alone prime-boost vaccination strategy or

18 as a boost for existing vaccines. 


\section{9}

20

21

22

23

24 simple logistic chain ${ }^{4,5}$.

25

\section{Introduction}

Current field observations show that a protective vaccine against SARS-CoV-2 is likely the only means of controlling the pandemic ${ }^{1-3}$. To fulfill this promise, these vaccines should ideally be effective in preventing infection and virus transmission and, importantly, well accepted by the population. In underdeveloped countries, vaccines should also have a

Regarding efficacy, years of vaccine research have demonstrated that vaccine protective effects rely in large part on systemic neutralizing antibodies, while local cytotoxic T cell responses are for the most part responsible for virus eradication after a productive infection ${ }^{6}$. Moreover, for upper respiratory tract infection, a robust mucosal immunity is likely required to minimize virus transmission ${ }^{7-9}$. Regarding vaccine hesitancy, oral administration would favor acceptance and minimize the risk of adverse events ${ }^{10}$. Regarding logistics, oral administration would also ease mass vaccination, and a thermostable vaccine would ensure a much-simplified logistic chain. Likewise, an optimal vaccine against SARS-CoV-2 should be thermostable, orally administered and able to induce a robust mucosal neutralizing immune response.

To tackle the challenge of producing such a vaccine, we used our platform based on retrovirus-derived enveloped virus-like particles (e-VLPs) that has been developed to generate neutralizing antibody $(N A b)^{11}$. Indeed, these e-VLPs have the same lipid membrane as the cell they derive from. Likewise, virus envelope proteins that VLPS express have the same conformation as they have on the lipid membrane of an infected cell, and on the virus itself. As NAbs are mostly targeted to conformational structures, e- 
41 VLPs are thus particularly suitable for NAb induction ${ }^{12}$. We previously showed that such e-

42 VLPs could generate robust NAbs against many viruses, such as influenza, HCV and CMV,

43 in mice, macaques and humans ${ }^{13-17}$. Moreover, exploiting the versatile engineering

44 possibilities for these e-VLPs, we recently showed that they could be harnessed with

45 variable surface proteins (VSPs) from the intestinal parasite Giardia lamblia, affording

46 them resistance to degradation and the triggering of robust mucosal cellular and antibody

47 immune responses after oral administration ${ }^{18,19}$.

48 We used this experience to design and evaluate a thermostable orally administered e-VLP

49 vaccine against SARS-CoV-2. We tested the expression of various forms of the Spike

50 protein (S) with or without SARS-CoV-2 membrane protein (M) expression. We found that

51 e-VLPs expressing a pre-fusion stabilized form of S plus M trigger robust mucosal NAbs

52 against SARS-CoV-2 in mice and hamsters, which translate into complete protection from

53 a viral challenge. We consider that such a vaccine could be part of the arsenal against

54 SARS-CoV-2, in a stand-alone prime-boost vaccination strategy or as a boost for existing

55 vaccines.

56

57 Materials and Methods

58 Viruses. SARS-CoV-2 isolates were propagated in Vero E6 cells in Opti-MEM I (Invitrogen,

59 Cat. \# 51985091) containing 0.3\% bovine serum albumin (BSA) and $1 \mu \mathrm{g}$ of L-1-tosylamide-

60 2-phenylethyl chloromethyl ketone-treated trypsin per $\mathrm{mL}$ at $37^{\circ} \mathrm{C}$. 
61 Experimental animals. For immunization and challenge, the group sizes were chosen

62 based on previous experience and littermates of the same sex were randomly assigned.

63 The number of animals for each experiment and all procedures followed the protocols

64 approved by the Institutional Committee for Care and Use of Experimental Animals. Six

65 week- or four month-old male and female BALB/c mice were used for initial experiments,

66 6-month-old female and male Golden Syrian hamsters were used in the immunization

67 studies, and 1-month-old female and male SPF Golden Syrian hamsters were used in the

68 challenge experiments. For challenge experiments, under ketamine-xylazine anesthesia,

69 ten hamsters per group were inoculated with $10^{5}$ PFU of SARS-CoV-2 (in $100 \mu \mathrm{L}$ ) or PBS

70 (mock) via the intranasal route ${ }^{20,21}$. Baseline body weights were measured before

71 infection and body weight was monitored for 28 days (Lee et al., 2020). No animals were

72 harmed during the collection of blood.

73 Neutralization assays. Serum taken from intranasally inoculated hamsters at 14 dpi was

74 tested for viral neutralizing antibody titer by microneutralizing assay in Vero E6 cells.

75 Briefly, dilutions of serum samples (1:50 to 1:10,000) were mixed with 100 TCID50 of

76 SARS-CoV-2 virus and incubated at $37^{\circ} \mathrm{C}$ for $1 \mathrm{~h}$. The mixture was then added to Vero E6

77 cells and further incubated at $37^{\circ} \mathrm{C}$ for $72 \mathrm{~h}^{22}$. The neutralizing antibody titer was defined

78 as the highest dilution that inhibits $50 \%$ of the cytopathic effect.

79 VLP expression plasmids. For pGag, the cDNA sequence encoding the MLV Gag (Uniprot:

80 PODOG8.1) without the C-terminal Pol sequence was used ${ }^{19}$. For SARS-CoV-2 spike protein

81 variants, the cDNA sequences were cloned in the phCMV expression vector ${ }^{19}$. All plasmids

82 were verified by sequencing as reported. The SARS-CoV-2 spike protein variants derived 
83 from the wild type strain Swt (NC_045512, original Wuhan variant) all having the D615G

84 and the 682RRAR-685GSAS (modFurinCS) mutations. Additional mutations were inserted

85 in specific variant: Sst1: K986P/V987P; Sst2: T791C/A879C; Sst3: S884C/A893C; Sst4:

86 G885C/Q913C; Sst5: S884C/Q913C.

87 VLP generation, production, purification, and validation. VLPS were produced by

88 transient transfection of either HEK293 cells or HEK293-1267 cells, with pGag, pS and its

89 variants, and pM plasmid DNA, using PEI as transfection reagent. Cells were transfected at

$9070 \%$ confluence in T175 flasks with $70 \mu \mathrm{g}$ of total DNA per flask at a PEI: DNA mass ratio of

$913: 1$. VLP-containing supernatants were harvested $72 \mathrm{~h}$ post-transfection, filtered through

92 a $0.45 \mu \mathrm{m}$-pore size membrane, and concentrated $20 \mathrm{x}$ in a centrifugal filter device

93 (Centricon ${ }^{\circledR}$ Plus-70-100 K, Millipore Cat. \# UFC710008) and purified by ultracentrifugation

94 through a $20 \%$ sucrose cushion in an SW41T Beckman rotor $\left(25,000 \mathrm{rpm}, 4 \mathrm{~h}\right.$, at $\left.4{ }^{\circ} \mathrm{C}\right)$.

95 Pellets were resuspended in sterile TNE buffer $(50 \mathrm{mM}$ Tris- $\mathrm{HCl}$ pH 7.4, $100 \mathrm{mM} \mathrm{NaCl}, 0.1$

$96 \mathrm{mM}$ EDTA). Proteins were measured using the Bradford method. For western blotting,

97 proteins were resolved by $10 \%$ SDS-PAGE and transferred onto PVDF membranes before

98 incubation with specific primary antibodies. Alkaline phosphatase-conjugated secondary

99 antibodies were used and were detected by BCIP/NBT substrate ${ }^{19}$.

100 Immunizations. Mice and Golden Syrian Hamsters were fasted for $4 \mathrm{~h}$ and then orally

101 immunized with two weekly doses of $100 \mu \mathrm{g}$ of different VLPs. For IM immunization, two

102 weekly doses of $10 \mu \mathrm{g}$ of different VLPs were administered. Animals from the negative

103 control group (naive) received oral immunizations with vehicle alone. 
104 Fluid collection. Blood was collected weekly from the retro-orbital sinus of hamsters and

105 serum was separated and stored at $-80^{\circ} \mathrm{C}$. BAL was collected through the trachea by

106 injection-aspiration of $1 \mathrm{~mL}$ of PBS with protease inhibitors.

107 Enzyme-linked immunosorbent assay (ELISA) tests. The levels of IgG and IgA antibodies

108 against spike protein were determined by ELISA by sensitizing the plate with homogenates

109 of killed whole virus produced in vitro. Spike was quantified using purified protein (Human

110 coronavirus HCoV-229E Spike Protein (S1+S2 ECD). Sino Biological, Inc. 40605-V08B and

111 Spike Protein (Active Trimer) R\&D Cat. \# 10549-CV). The following secondary antibodies

112 were used: Mouse anti-Hamster IgG Cocktail, Clone: G94-56, G70-204 (BD Biosciences,

113 Cat. \# 554009); Mouse anti-Hamster IgM, Clone: G188-9, (BD Biosciences Cat. \# 554035);

114 Hamster Immunoglobulin A (IgA) ELISA Kit (MyBiosources Cat. \# MBS029668); Mouse

115 monoclonal (H6) anti-SARS-CoV-2 spike glycoprotein (Abcam Cat. \# ab273169).

116 Statistical analyses. Prism (GraphPad Software) was used to perform one-way or two-way

117 ANOVA on datasets with Tukey's multiple comparisons test or the Bonferroni post-test,

118 respectively. All figures show mean \pm S.E.M. Statistically significant differences are

119 indicated in each graph as ${ }^{*} p<0.05,{ }^{* *} p<0.01$ and ${ }^{* * *} p<0.001$ and ns = not significant. 


\section{Results}

122 Designing and selecting the immunogens.

123 Initially, the spike protein S of SARS-CoV-2 was evaluated and several variants for

124 stabilization of the receptor-binding domain (RBD) and stabilization in the pre-fusion state

125 were designed (Fig.1A). Point mutations, Cys-molecular clamps, furin-cleavage site

126 elimination, and Proline (Pro) substitutions ${ }^{23,24}$ were generated and cloned. Spike protein

127 variants that conserved their own cytoplasmic tail (CT), although it can be advantageous

128 to swap it for the CT of VSV-G to improve pseudotyping onto eVLPs, or that were modified

129 to delete its ER retention signal were also designed. Then, those VLPs were produced and

130 validated for the correct composition as described ${ }^{25}$. VSP-pseudotyped VLPs were orally

131 administered to BALB/c mice and the level of serum IgGs was determined by ELISA (Fig. 2).

132 Notably, spike variants lacking the furin-cleaved site and with the two Pro substitutions

133 (Sst1), as reported by Wrapp et al. $(2020)^{26}$, including the D614G mutation ${ }^{27,28}$, was the

134 most efficient in eliciting a high level of antibodies. Identical experiments were performed

135 in ob/ob mice (JAX ${ }^{\mathrm{TM}}$ Mice Strain), $\mathrm{db} / \mathrm{db}$ mice (JAX ${ }^{\mathrm{TM}}$ Mice Strain) and aged mice ( $>4$

136 month-old) of both sexes and yielded similar results (not shown), indicating that antibody

137 production after oral immunization with e-VLPs does not vary according to underlying

138 condition, sex or age of the mice. Therefore, Sst1, the most effective variant of S, was

139 selected for further studies.

140 Given that glycosylation of spike would be important for its appropriate conformation

141 since there are numerous glycosylated sites near the RDB ${ }^{29,30}$, glycosylation of S should 
142 likely influence the generation of efficient NAbs. As the C-terminal CT of the SARS-CoV-2

143 spike is important in proper glycosylation, spike with a CT modified to abolish ER retention

144 (SmodCT) was generated. In addition, the envelope membrane protein $M$ is known to

145 retain S at the ER for improvement of the first steps of glycosylation and, subsequently,

146 remains attached to the CT of S during their journey throughout the Golgi apparatus,

147 where final glycosylation is accomplished ${ }^{31}$. Consequently, e-VLPs with protein M of SARS-

148 CoV-2 were also tested (Fig. 1B). Although that was the main reason for including $M$ in the

149 e-VLPs, subsequent reports showed a specific T cell response to several epitopes of this

150 protein in patients who recovered from COVID-19 $9^{32-34}$. Thus, incorporation of $M$ in the

151 envelope of the e-VLPs could not only benefit proper glycosylation of S, but also the

152 production of a stronger cellular response to the virus. Therefore, e-VLPs with or without

$153 \mathrm{M}$ and with and without a modified CT were generated. Finally, all these e-VLPs were

154 generated with or without the incorporation onto the VLP surface of a VSP derived from

155 Giardia $^{25,35}$

156 e-VLP immunogenicity after intramuscular injections.

157 e-VLPs administered intramuscularly (i.m.) to hamsters, whether or not decorated with

158 VSPs, induced high levels of IgG (Fig. 3, left) and moderate level of IgA (Fig. 3, right) in

159 serum, validating these immunogens. Noteworthy, the presence of the Giardia VSP on the

160 e-VLPs promoted higher levels of antibodies than the plain e-VLP, highlighting the

161 adjuvant effect of the VSPs ${ }^{25,35}$.

162 The levels of serum IgG after immunization with Sst1 were higher than with the wild-type 163 spike, and the addition of $M$ further increased the IgG response. The levels of serum IgGs 
164 were also higher than those of IgA were, as expected. However, these values are

165 comparable to if not higher than those obtained after immunization with other vaccine

166 formulations ${ }^{36,37}$ or those found in plasma from convalescent patients ${ }^{38,39}$.

167 Altogether, these results clearly show that plain e-VLPs are already good immunogens

168 when administered by injection in the absence of any adjuvant, highlighting that VLPs are

169 structures well recognized by the immune system. These responses are strongly increased

170 when VSPs were present on e-VLPs according to the VSPs' intrinsic TLR4-dependent

171 adjuvant properties ${ }^{25,35}$.

e-VLP immunogenicity after oral administration.

173 Oral administration of the same validated immunogens showed that the absence of the

174 Giardia VSP decorating the different e-VLPs led to no detectable immune response, most

175 likely due to destruction of the VLPs in the upper small intestine (Fig. 4). Additionally, the

176 modification of the CT of S appeared detrimental in inducing either serum IgG (Fig. 4 left)

177 or IgA (Fig. 4 right) as compared with S having the wild-type CT. However, serum IgG and

178 IgA titers were augmented when M was incorporated into the VSP-e-VLPs (Fig. 4).

179 Noteworthy, the serum IgA induced by the VSP-decorated e-VLPs were higher after oral

180 administration (Fig. 4 right) than after i.m. injections (Fig. 3 right).

181 Altogether, these results show that the VSPs are essential for oral immunization with VLPS

182 and the immunogenicity of orally administered e-VLPS was strictly dependent on the

183 presence of the VSP on their surface, highlighting the efficiency of this route of 
184 immunization, which provides slightly higher Ig responses than after i.m. injection of the

185 same immunogens.

e-VLP-induced mucosal immunity.

187 When the presence of IgA was analyzed in bronchoalveolar lavages (BAL) of animals

188 immunized orally or by i.m. injection, it was noticed that intramuscularly immunized

189 animals have a consistently low level of IgA titers as compared with those administered

190 orally, in which only the e-VLP version containing VSP, M, and stabilized S showed high

191 titers of IgA (Fig. 5). Again, these results confirm (i) the high immunogenicity of e-VLP

192 formulations, (ii) the crucial role of VSPs in protection of e-VLPs, and (iii) the higher

193 efficiency of oral administration in inducing mucosal IgA.

\section{$194 \quad$ e-VLP-induced neutralizing antibodies.}

195 We then compared the best e-VLPs expressing the Sst1 spike and M proteins with the e-

196 VLPs expressing a wild-type spike for the generation of neutralizing antibodies.

197 Interestingly, there was no NAb generated after i.m. injection when the VSP was not

198 present on the VLPs (Fig. 6), highlighting its adjuvant effect. With the VSPs present, both

199 wild-type and stabilized S was able to generate NAbs, as observed for the different

200 commercial vaccines already being administered to humans ${ }^{1,40-42}$. Remarkably, the titer of

201 NAbs generated after oral administration are equivalent to those generated after i.m.

202 injections (Fig. 6), highlighting the efficiency of VSP-e-VLPs as immunogens. 
204 Given the incomplete level of protection afforded by some vaccines and the constant

205 emergence of new viral variants, these orally administered immunogens could possibly be

206 good boosts for existing vaccines. For these reasons, an oral boost was applied to animals

207 previously vaccinated by i.m. injections. In these animals, a third dose of the oral

208 formulation containing VSP, stabilized S and M induced a major increase in the levels of

209 IgA in BAL as compared to those that only received two doses intramuscularly (Fig. 7).

\section{Oral vaccination with VSP-e-VLPs protects hamsters from a challenge with SARS-CoV-2.}

211 Animals immunized with e-VLPs in which stabilized S and M were present on the particles

212 with or without VSP pseudotyping were challenged with SARS-CoV-2 and the clinical

213 response of the hamsters was determined by monitoring their weight ${ }^{21}$. Control animals

214 lost weight during the two weeks following the viral challenge (Fig. 8) and then recovered,

215 as reported for experimental infections in hamsters ${ }^{20,21}$. Hamsters that were only

216 immunized intramuscularly were not fully protected as they had only a slightly lower

217 weight loss. In contrast, oral immunization with VSP-e-VLPs fully prevented weight loss,

218 whether or not the M protein was present, and similarly to animals that were immunized

219 by injection first and then boosted orally (Fig. 8).

\section{Discussion}

222 The differences observed between the same formulations administered either orally or

223 intramuscularly in these animals suggest that although the oral route is expected to show

224 a higher degree of variation among animals, this was not the case. This could be explained 
225 by the type of generated Igs. Notably, considering the i.m. administration was done in the

226 absence of any added adjuvant, the high immunogenicity of VSP-e-VLPs can be explained

227 by the adjuvant properties of the VSPs, which have been demonstrated to activate TLR-

$2284^{19}$. The immunogenicity of the e-VLPs lacking VSPs may mainly rely on the particulate

229 nature of VLPS and the repetitive exposure of the antigen on their surface, even TLR-

230 signaling as been described ${ }^{43}$.

231 Our results first show that it is possible to co-express SARS-CoV-2 envelope proteins

232 together with Giardia VSPs on e-VLPs to generate mucosal Igs and NAbs against SARS-CoV-

2332 after oral administration. While plain e-VLPs did not generate any Ab responses, VSP-

234 decorated e-VLPs (VSP-e-VLPs) generated Ab responses in the range of, if not higher than,

235 the response to i.m. administration. This is remarkable as it indicates that the SARS-CoV-2

236 Env proteins are well protected from degradation by VSPs as they preserve their proper

237 conformation, which is needed for NAb production. Thus, this extends our previous results

238 with e-VLP expressing HA of influenza ${ }^{19}$, demonstrating the versatility of the VSP-e-VLP

239 platform. Actually, the dual properties of VSPs were confirmed: they not only afford

240 protection from degradation, but also have a potent adjuvant effect. Indeed, when

241 vaccines are administered i.m., VSP-e-VLP always led to higher titers of antibodies than

242 their plain e-VLPs. Of note, a SARS-CoV-2 VLP based on our platform technology ${ }^{15}$ was

243 independently reported to generate a good NAb response after i.m. administration, but

244 with no reports of IgA at mucosal sites.

245 Besides its ease, oral administration is known for also having the advantage of triggering

246 better mucosal immunity. This is indeed the case here, with high levels of plasma but also 
247 bronchoalveolar lavage IgA detectable only after oral administration. This is an obvious

248 advantage for a vaccine against SARS-CoV-2, as it should reduce viral transmission. In this

249 line, SARS-CoV-2 was still detected in BAL of i.m. vaccinated macaques that otherwise

250 appeared protected from infection. Whether a better mucosal response, as afforded by

251 VSP-e-VLPS, will completely sterilize challenged macaques requires further investigation.

252 We have not tested the specific T cell response in this study. However, it is known that e-

253 VLPs do induce robust cellular responses; indeed, using VSP-HA-VLPs, a strong cytotoxic T

254 lymphocyte response was generated that was able to kill HA-expressing tumor cells ${ }^{25}$.

255 Moreover, the IgG and IgA responses here are notoriously T cell-dependent and the good

256 antibody response thus attests to a good T cell response ${ }^{44}$. In this line, we previously

257 showed that the fusion of a viral peptide to Gag, the retroviral protein precursor that

258 drives the formation and release of the viral particle/VLPs, produces additional strong T

259 cell responses against this peptide ${ }^{12}$. The fusion to Gag of large fragments or the SARS-

260 CoV-2 N structural protein, or a stretch of immunodominant and/or conserved peptides,

261 would be a mean to further enhance the immunogenicity of VSP-e-VLPs and enhance

262 protection against variants.

263 SARS-CoV-2 e-VLPS and VSP-e-VLPs could be used as a stand-alone vaccine, likely with a

264 prime-boost scheme of administration. VSP-e-VLPs are thermostable ${ }^{19}$, retaining their

265 properties at room temperature and tolerating several freeze-thaw cycles, and could thus

266 be particularly advantageous for vaccination in countries where refrigeration of vaccine

267 supplies is problematic. VSP-e-VLPs could also be used as a boost for other vaccine

268 designs. In this regard, it is still unknown how long the protection afforded by the 
269 currently used vaccines will last. The follow-up of infected patients indicates that, at least

270 for some patients, the persistence of NAbs and the duration of protection might last a few

271 months ${ }^{45}$. These findings, plus the advent of viral variants, make it likely that the global

272 population will need to boost the immune response of vaccinees regularly. For some

273 vaccine designs, and particularly those based on adenoviral vectors, the re-administration

274 of the same vector might not be very efficient due to the immune response generated

275 against the vector. For these, a boost with VSP-e-VLPs might be particularly interesting.

276 For other vaccine designs, and especially if repeated administrations are needed over the

277 years, an orally administered vaccine might be more acceptable.

278 The SARS-CoV-2 pandemic calls for vaccination of very large groups of people. This

279 requires a suitable production of vaccine with an excellent safety profile. Noteworthy, we

280 contributed to the design of an anti-CMV e-VLP vaccine based on our e-VLP platform that

281 has already been used in patients, demonstrating scalable GMP production and an

282 excellent safety profile $\mathrm{e}^{15,17,46}$.

283 Altogether, given the specific issues of each vaccine design (thermostability, side effects,

284 lack of mucosal immunity induction, immunogenicity against the vector, among other

285 benefits), the availability of multiple vaccines against SARS-CoV-2 improves our chances of

286 controlling the pandemic. In this regard, a thermostable orally administered e-VLP vaccine

287 will be a valuable addition to the current arsenal against this virus. 


\section{References}

289

290

291

292

293

294

295

296

297

298

299

300

301

302

303

304

305

306

307

308

309

310

311

312

313

314

315

316

317

318

319

320

321

322

323

324

325

326

327

328

329

1. Becker, M. et al. Immune response to SARS-CoV-2 variants of concern in vaccinated individuals. Nat Commun 12, 3109 (2021).

2. Saad-Roy, C. M. et al. Immune life history, vaccination, and the dynamics of SARSCoV-2 over the next 5 years. Science 370, 811-818 (2020).

3. Sultana, J. et al. Potential effects of vaccinations on the prevention of COVID-19: rationale, clinical evidence, risks, and public health considerations. Expert Rev Vaccines 19, 919-936 (2020).

4. Dube, E., Vivion, M. \& MacDonald, N. E. Vaccine hesitancy, vaccine refusal and the anti-vaccine movement: influence, impact and implications. Expert Rev Vaccines 14, 99117 (2014).

5. Salmon, D. A., Dudley, M. Z., Glanz, J. M. \& Omer, S. B. Vaccine Hesitancy: Causes, Consequences, and a Call to Action. Am J Prev Med 49, S391-8 (2015).

6. Pollard, A. J. \& Bijker, E. M. A guide to vaccinology: from basic principles to new developments. Nat Rev Immunol 21, 83-100 (2021).

7. Brandtzaeg, P. Induction of secretory immunity and memory at mucosal surfaces. Vaccine 25, 5467-84 (2007).

8. Holmgren, J. \& Czerkinsky, C. Mucosal immunity and vaccines. Nat Med 11, S45-53 (2005).

9. Lavelle, E. C. \& Ward, R. W. Mucosal vaccines - fortifying the frontiers. Nat Rev Immunol (2021) doi:10.1038/s41577-021-00583-2.

10. Wang, S., Liu, H., Zhang, X. \& Qian, F. Intranasal and oral vaccination with proteinbased antigens: advantages, challenges and formulation strategies. Protein Cell 6, 480-503 (2015).

11. Pitoiset, F., Vazquez, T. \& Bellier, B. Enveloped virus-like particle platforms:

Vaccines of the future? Expert Rev Vaccines 1-3 (2015)

doi:10.1586/14760584.2015.1046440.

12. Bellier, B. \& Klatzmann, D. Virus-like particle-based vaccines against hepatitis C virus infection. Expert Rev Vaccines 12, 143-154 (2013).

13. Bellier, B. et al. DNA vaccines expressing retrovirus-like particles are efficient immunogens to induce neutralizing antibodies. Vaccine 27, 5772-80 (2009).

14. Dalba, C., Bellier, B., Kasahara, N. \& Klatzmann, D. Replication-competent vectors and empty virus-like particles: new retroviral vector designs for cancer gene therapy or vaccines. Mol Ther 15, 457-66 (2007).

15. Fluckiger, A.-C. et al. An enveloped virus-like particle vaccine expressing a stabilized prefusion form of the SARS-CoV-2 spike protein elicits potent immunity after a single dose. http://biorxiv.org/lookup/doi/10.1101/2021.04.28.441832 (2021) doi:10.1101/2021.04.28.441832.

16. Garrone, P. et al. A prime-boost strategy using virus-like particles pseudotyped for HCV proteins triggers broadly neutralizing antibodies in macaques. Sci Transl Med 3, 94ra71 (2011).

17. Kirchmeier, M. et al. Enveloped virus-like particle expression of human 
cytomegalovirus glycoprotein B antigen induces antibodies with potent and broad neutralizing activity. Clin Vaccine Immunol 21, 174-180 (2014). 18. Rupil, L. L., Serradell, M. del C. \& Luján, H. D. Using Protozoan Surface Proteins for Effective Oral Vaccination. Trends in Parasitology 36, 7-10 (2020). 19. Serradell, M. C. et al. Efficient oral vaccination by bioengineering virus-like particles with protozoan surface proteins. Nat Commun 10, 361 (2019). 20. Imai, M. et al. Syrian hamsters as a small animal model for SARS-CoV-2 infection and countermeasure development. Proc Natl Acad Sci USA 202009799 (2020) doi:10.1073/pnas.2009799117.

21. Lee, A. C.-Y. et al. Oral SARS-CoV-2 Inoculation Establishes Subclinical Respiratory Infection with Virus Shedding in Golden Syrian Hamsters. Cell Reports Medicine 1, 100121 (2020).

22. Chen, F. et al. In vitro susceptibility of 10 clinical isolates of SARS coronavirus to selected antiviral compounds. J Clin Virol 31, 69-75 (2004). 23. Bangaru, S. et al. Structural analysis of full-length SARS-CoV-2 spike protein from an advanced vaccine candidate. Science 370, 1089-1094 (2020). 24. Hsieh, C.-L. et al. Structure-based design of prefusion-stabilized SARS-CoV-2 spikes. Science 369, 1501-1505 (2020).

25. Serradell, M. C. et al. Efficient oral vaccination by bioengineering virus-like particles with protozoan surface proteins. Nat Commun 10, 361 (2019). 26. Wrapp, D. et al. Cryo-EM structure of the 2019-nCoV spike in the prefusion conformation. Science 367, 1260-1263 (2020).

352 27. Korber, B. et al. Tracking Changes in SARS-CoV-2 Spike: Evidence that D614G 353 Increases Infectivity of the COVID-19 Virus. Cell 182, 812-827.e19 (2020).

354 28. Plante, J. A. et al. Spike mutation D614G alters SARS-CoV-2 fitness. Nature 592, 355 116-121 (2021).

356 29. Grant, O. C., Montgomery, D., Ito, K. \& Woods, R. J. Analysis of the SARS-CoV-2 spike protein glycan shield reveals implications for immune recognition. Sci Rep 10, 14991

358 (2020).

359 30. Watanabe, Y., Allen, J. D., Wrapp, D., McLellan, J. S. \& Crispin, M. Site-specific glycan analysis of the SARS-CoV-2 spike. Science (2020).

361 31. Boson, B. et al. The SARS-CoV-2 envelope and membrane proteins modulate maturation and retention of the spike protein, allowing assembly of virus-like particles. $J$ Biol Chem 296, 100111 (2021).

364 32. Braun, J. et al. SARS-CoV-2-reactive T cells in healthy donors and patients with COVID-19. Nature 587, 270-274 (2020). with COVID-19 Disease and Unexposed Individuals. Cell 181, 1489-1501.e15 (2020). unexposed humans. Science 370, 89-94 (2020).

370 35. Rupil, L. L., Serradell, M. D. C. \& Luján, H. D. Using Protozoan Surface Proteins for 371 Effective Oral Vaccination. Trends Parasitol 36, 7-10 (2020).

372 36. Chi, X. et al. A potent neutralizing human antibody reveals the N-terminal domain 373 of the Spike protein of SARS-CoV-2 as a site of vulnerability. 
374 http://biorxiv.org/lookup/doi/10.1101/2020.05.08.083964 (2020)

375 doi:10.1101/2020.05.08.083964.

376 37. Lau, E. H. Y. et al. Neutralizing antibody titres in SARS-CoV-2 infections. Nat

377 Commun 12, 63 (2021).

378 38. Cao, Y. et al. Potent Neutralizing Antibodies against SARS-CoV-2 Identified by High-

379 Throughput Single-Cell Sequencing of Convalescent Patients' B Cells. Cell 182, 73-84.e16

380 (2020).

381 39. Sekine, T. et al. Robust T Cell Immunity in Convalescent Individuals with

382 Asymptomatic or Mild COVID-19. Cell 183, 158-168.e14 (2020).

383 40. Dong, Y. et al. A systematic review of SARS-CoV-2 vaccine candidates. Sig Transduct

384 Target Ther 5, 237 (2020).

385 41. Hassan, A. O. et al. A Single-Dose Intranasal ChAd Vaccine Protects Upper and

386 Lower Respiratory Tracts against SARS-CoV-2. Cell 183, 169-184.e13 (2020).

38742 Isakova-Sivak, I. \& Rudenko, L. A promising inactivated whole-virion SARS-CoV-2

388 vaccine. The Lancet Infectious Diseases 21, 2-3 (2021).

389 43. Pitoiset, F. et al. Retrovirus-Based Virus-Like Particle Immunogenicity and Its

390 Modulation by Toll-Like Receptor Activation. J. Virol. 91, (2017).

391 44. Wang, Z. et al. Enhanced SARS-CoV-2 Neutralization by Secretory IgA in vitro.

392 http://biorxiv.org/lookup/doi/10.1101/2020.09.09.288555 (2020)

393 doi:10.1101/2020.09.09.288555.

394 45. Hodgson, S. H. et al. What defines an efficacious COVID-19 vaccine? A review of 395 the challenges assessing the clinical efficacy of vaccines against SARS-CoV-2. Lancet Infect 396 Dis 21, e26-e35 (2021).

397 46. Plotkin, S. A. et al. The Status of Vaccine Development Against the Human

398 Cytomegalovirus. J Infect Dis 221, S113-S122 (2020). 
401 Acknowledgments: This work was supported by grants from FONCYT (PICT-E 0234, and

402 PICT-2116), CONICET (D4408), and UCC (80020150200144CC) of Argentina, a Georg

403 Forster Award of the Alexander von Humboldt Foundation of Germany to H.D.L., and

404 recurrent funding by Sorbonne University and INSERM to DK.

405 Author contributions: HDL \& DK conceived the project. BB, HDL \& DK designed the

406 experiments, and supervised students and technicians. AS, LL, CM performed

407 experiments. BB, HDL and DK wrote the paper.

408 Competing interests: HL and DK are inventors of a patent application claiming orally

409 administered vaccines against coronaviruses that is owned by their public institutions. 


\section{Figures}

A

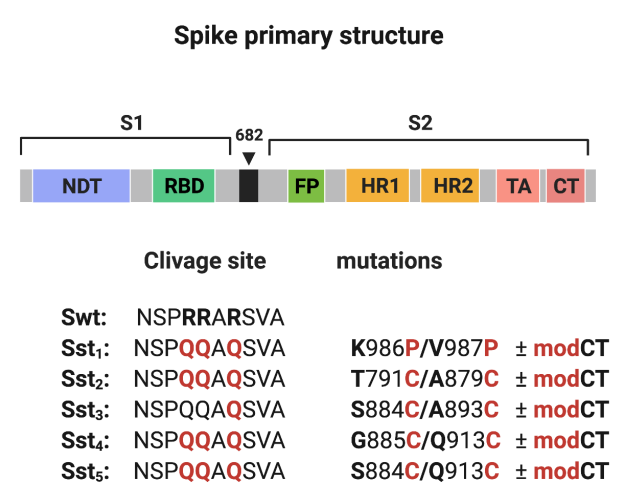

B

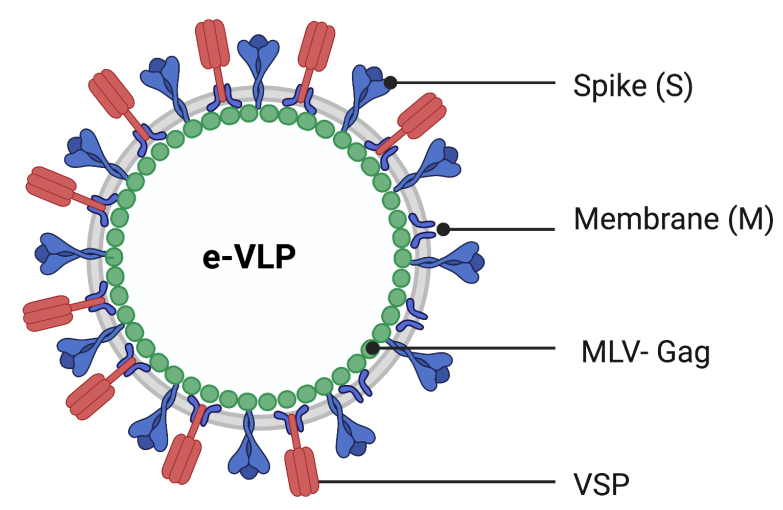

412

413 Figure 1. Structure and organization of the SARS-CoV-2 vaccine.

414 (A) Linear diagram of the sequence and structure elements of the SARS-CoV-2 spike

415 proteins used as immunogens. Structural elements include the S1 and S2 ectodomains

416 derived from the original Wuhan variant (Swt) in which specific mutation were inserted.

417 The native furin cleveage site was mutated (RRAR $\rightarrow$ QQAQ) in all variants ( Sst $_{1}-$ Sst $_{5}$ ) to be

418 protease resistant. Specific subsitution (in red) and respective position were indicated.

419 The spike variants with a CT modified to abolish ER retention (modCT) were also

420 generated. (B) SARS-CoV-2 eVLP structure. Native or mutated form of the SARS-CoV-2

421 spike (S) were pseudotyped on VLP formed with the viral matrix protein MLV-Gag in

422 association or not with the SARS-CoV-2 M proteins and the VSP from the intestinal

423 parasite Giardia lamblia. 


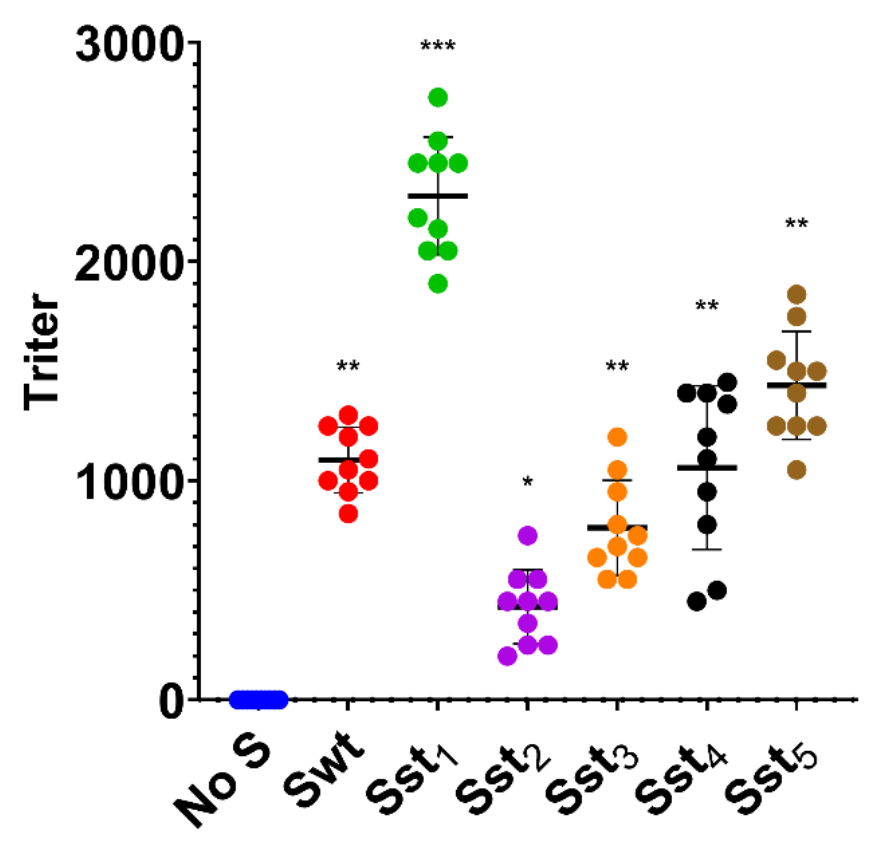

426 Figure 2. Immunogenicity of different variants of SARS-CoV-2 spike-e-VLPs in mice after

427 oral immunization. VSP-pseudotryped e-VLPs displaying the following SARS-CoV-2 spike

428 protein variants (Sst1, Sst2, Sst3, Sst4, Sst5) or wilt type sequence (Swt) were produced

429 and used for oral immunization as described in Methods. No S means e-VLPs without

430 spike. Values represent the IgG titer in blood of each animal and the horizontal line

431 indicates the mean value. Stabilized Spike 1 (Sst1) presented the higher titers and was

432 selected for subsequent experiments. 

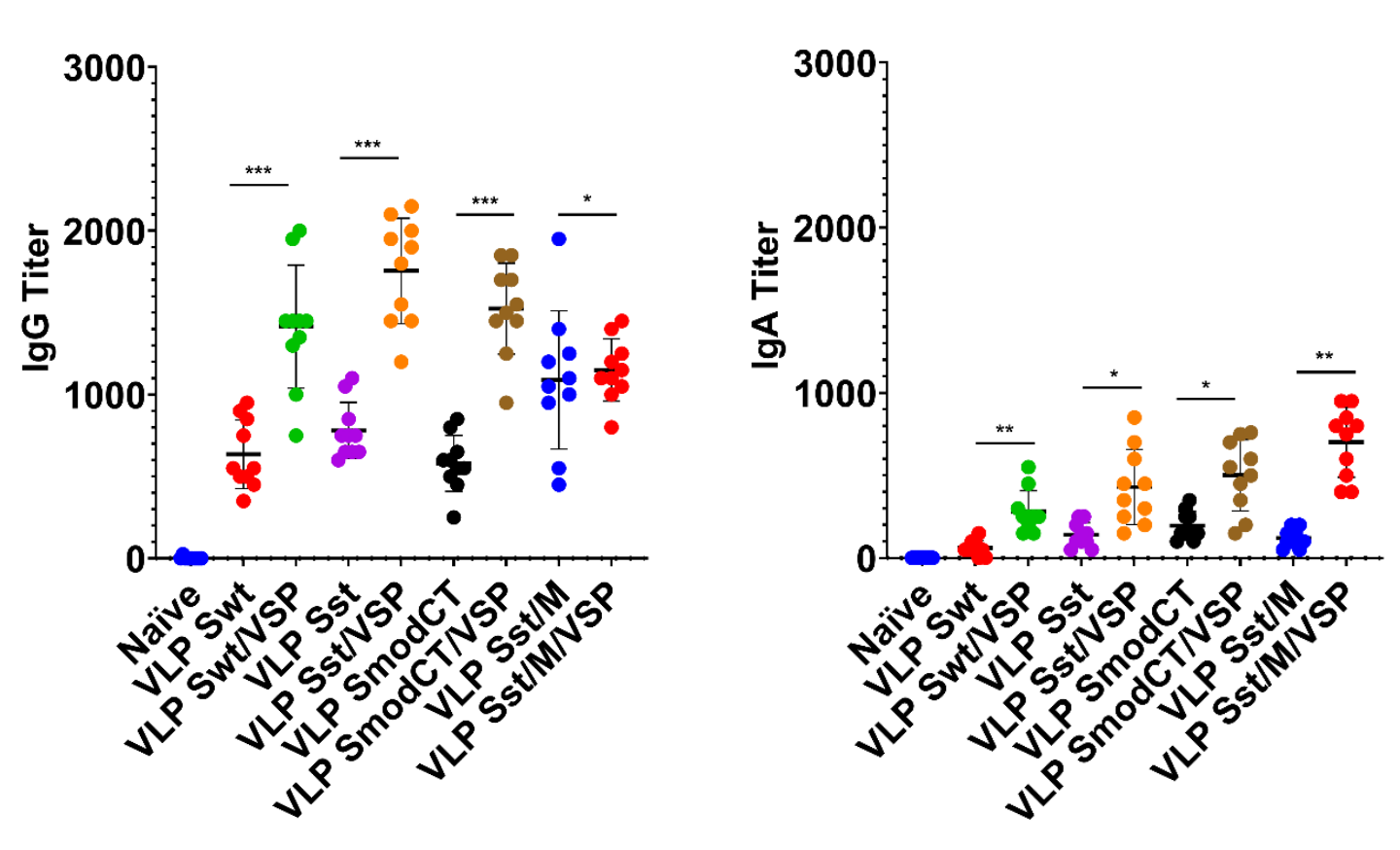

435 Figure 3. Serum antibody responses to intramuscular administration of different vaccine

436 formulations in hamsters. Serum IgG (left) and serum IgA (right) titers of hamsters

437 unvaccinated (naïve) or vaccinated intramuscularly with different formulations. Wild-type

438 spike (Swt), stabilized S as in Fig. 1 (Sst) and the same in which the cytoplasmic tail was

439 modified (SmodCT) were used to pseudotype e-VLPs including or not SARS-CoV-2 M

440 protein and Giardia VSPs. Data were analyzed by one-way ANOVA and Sidak's comparison

441 test. Values represent the mean \pm s.e.m. ${ }^{*} p<0.05 ;{ }^{* *} p<0.01 ;{ }^{* * *} p<0.001$; ns, not

442 significant. 

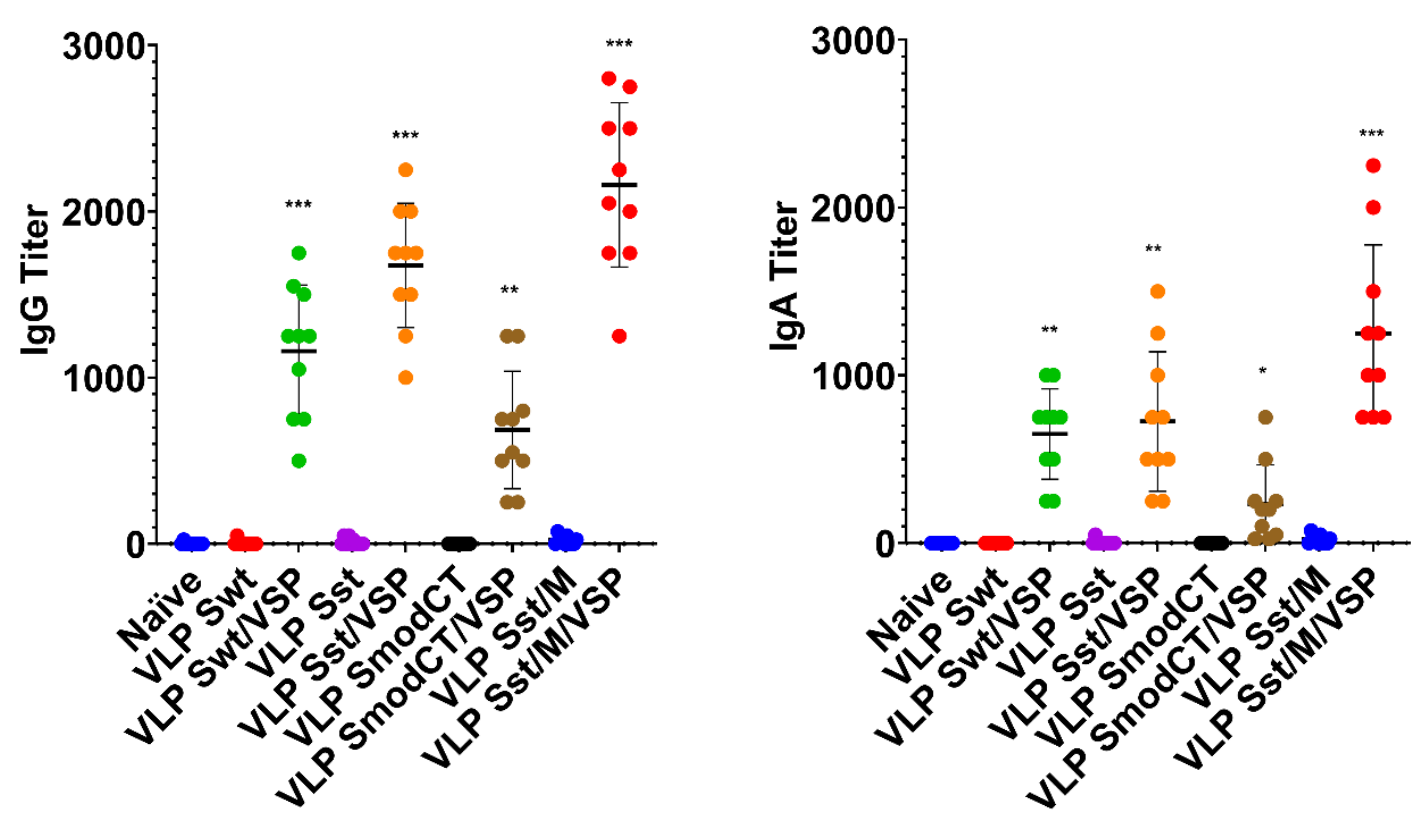

445 Figure 4. Serum antibody responses to oral administration of different vaccine

446 formulations in hamsters. Serum IgG (left) and serum IgA (right) titers of hamsters

447 unvaccinated (naïve) or vaccinated orally with different formulations. Wild-type spike

448 (Swt), stabilized S as in Fig. 1 (Sst) and the same in which the cytoplasmic tail was modified

449 (SmodCT) were used to pseudotype e-VLPs including or not SARS-CoV-2 M protein and

450 Giardia VSPs. Data were analyzed by one-way ANOVA and Sidak's comparison test. Values

451 represent the mean \pm s.e.m. ${ }^{*} p<0.05 ;{ }^{* *} p<0.01 ;{ }^{* *} p<0.001 ;$ ns, not significant. 

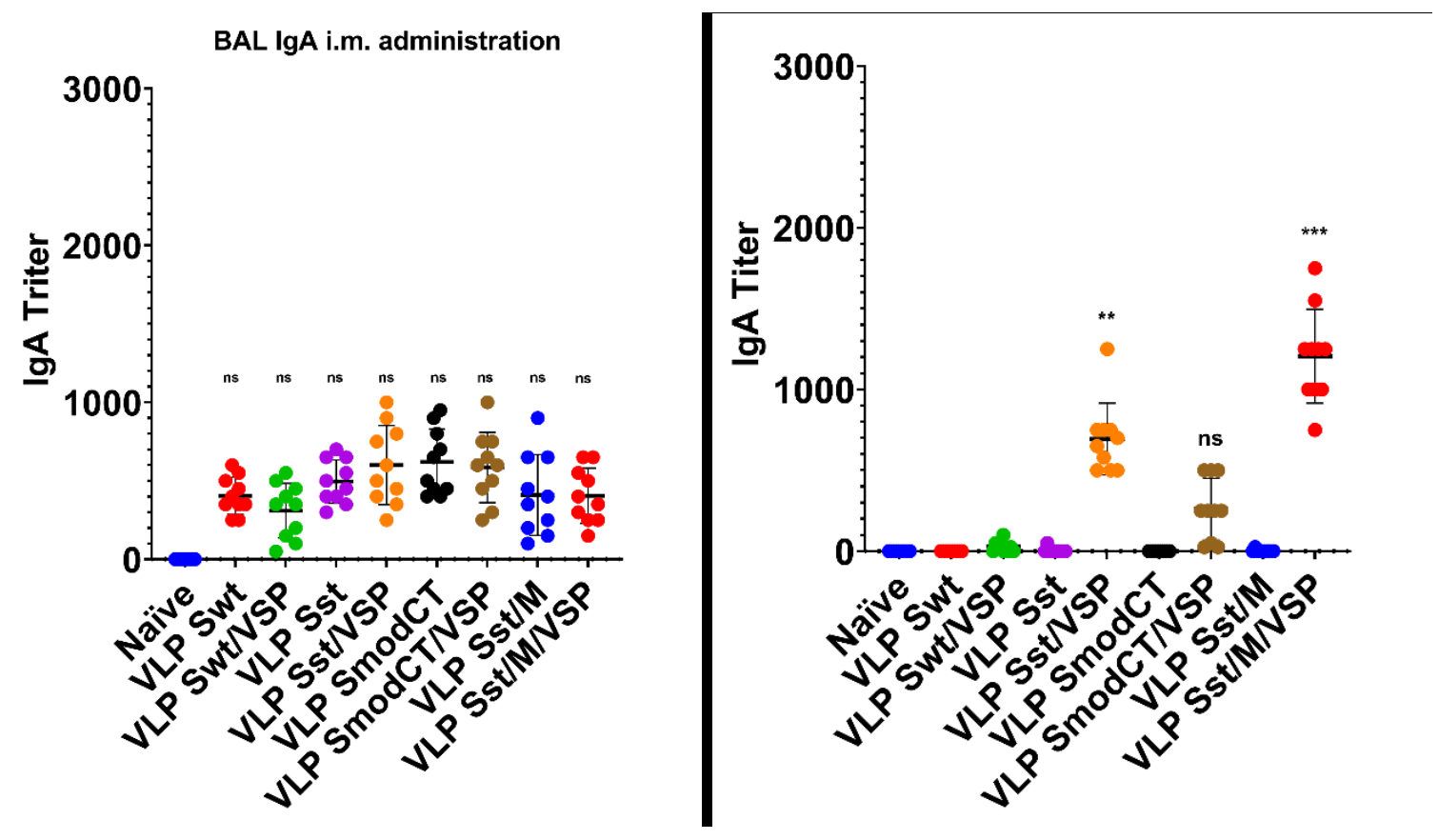

454 Figure 5. Bronchoalveolar lavage IgA responses. Bronchoalveolar lavage IgA titers of

455 hamsters unvaccinated (naïve) or vaccinated intramuscularly (left) or orally (right) with

456 different formulations. Wild-type spike (Swt), stabilized S as in Fig. 1 (Sst) and the same in

457 which the cytoplasmic tail was modified (SmodCT) were used to pseudotype e-VLPS

458 including or not SARS-CoV-2 M protein and Giardia VSPs. Data were analyzed by one-way

459 ANOVA and Sidak's comparison test. Values represent the mean \pm s.e.m. ${ }^{*} p<0.05$;

460

${ }^{* *} p<0.01 ; * * * p<0.001 ; \mathrm{ns}$, not significant. 


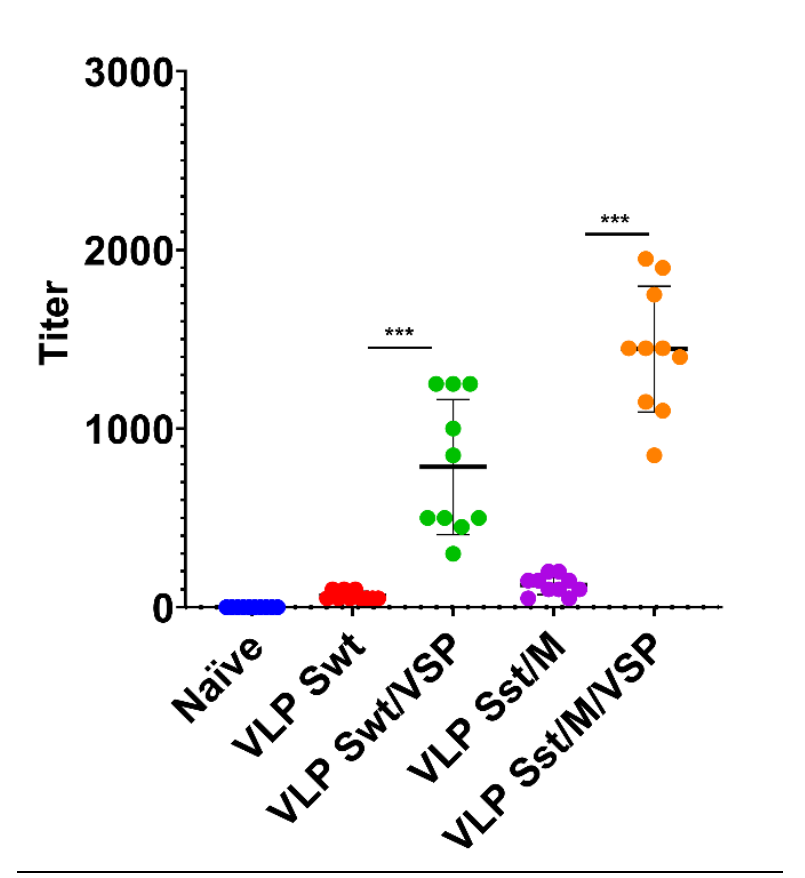

463

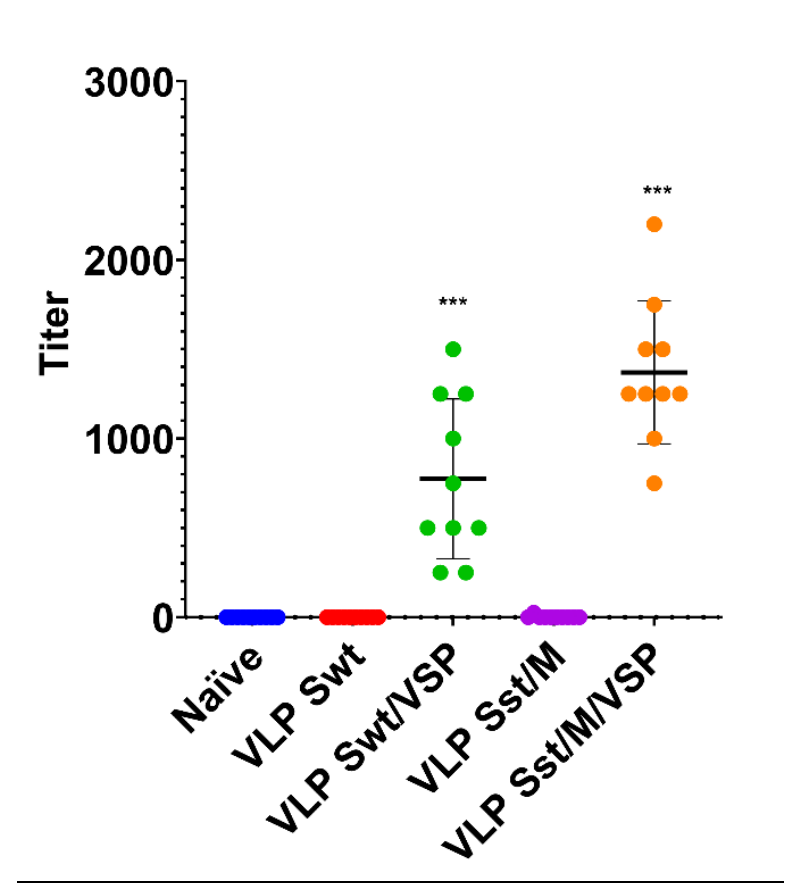

464 Figure 6. Neutralizing antibodies against SARS-CoV-2 entry. Neutralizing antibody titers

465 of intramuscularly (left) and orally (right) vaccinated animals with selected e-VLP

466 formulations and control animals (naïve). Data between e-VLPs pseudotyped or not with 
bioRxiv preprint doi: https://doi.org/10.1101/2021.09.09.459634; this version posted September 9, 2021. The copyright holder for this preprint (which was not certified by peer review) is the author/funder. All rights reserved. No reuse allowed without permission.

467 Giardia VSPs were analyzed by one-way ANOVA and Sidak's comparison test. Values

468 represent the mean \pm s.e.m. ${ }^{*} p<0.05 ;{ }^{*} p<0.01 ; * * p<0.001 ;$ ns, not significant. 


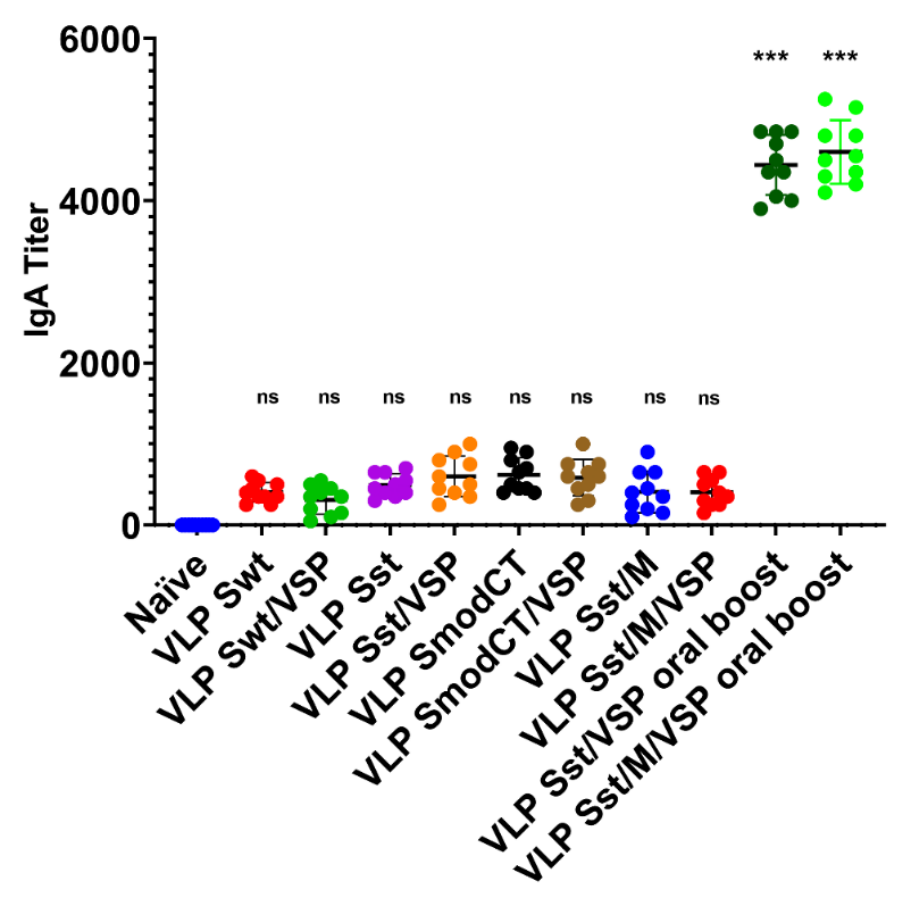

471 Figure 7. Bronchoalveolar IgA after an oral boost of intramuscularly vaccinated

472 hamsters. Same as Fig. 4a but including an extra oral boost with formulation including

473 stabilized spike and VSP in the presence or absence of SARS-CoV-2 M protein. Data were

474 analyzed by one-way ANOVA and Sidak's comparison test. Values represent the mean \pm

475 S.E.M. ${ }^{*} p<0.05 ;{ }^{* *} p<0.01 ;{ }^{* * *} p<0.001 ;$ ns, not significant. 


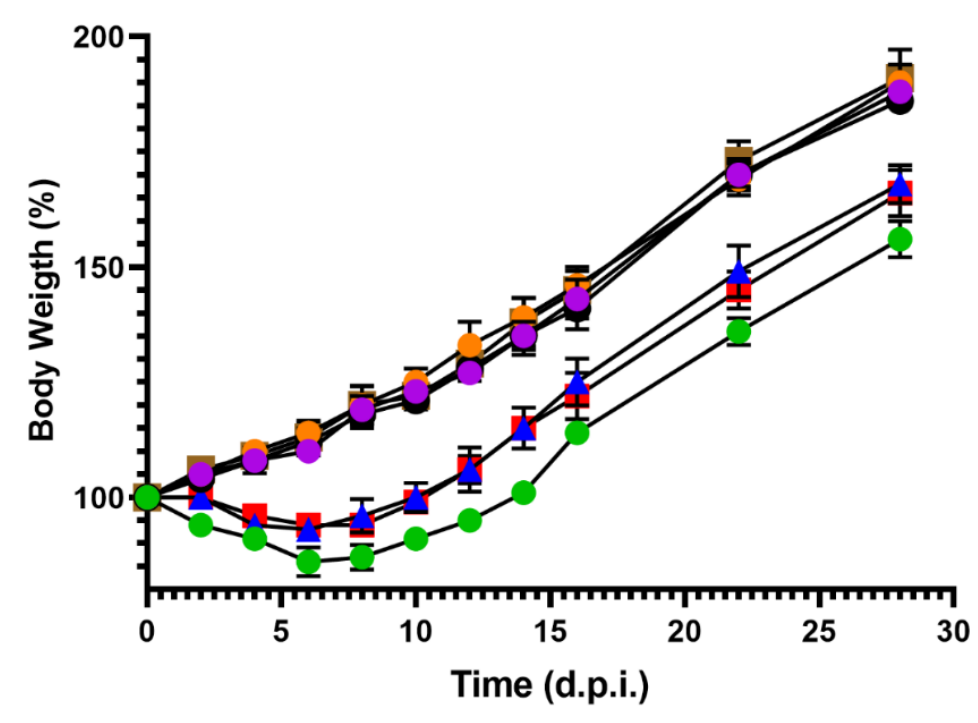

477

478 Figure 8. Vaccinated hamsters challenged with SARS-CoV-2. Hamsters either orally or

479 intramuscularly vaccinated were challenged intranasally with purified SARS-CoV-2 virus.

480 Every two days, their weight and general status were monitored and recorded.

481 Unvaccinated animals (green circles); Hamsters orally vaccinated with e-VLPs expressing

482 VSP and stabilized S with the addition or not of the M protein (purple and yellow circles

483 respectively); Intramuscularly vaccinated animals with the same formulations (blue

484 triangles, red squares); oral boost of i.m. vaccinated animals (black circles, brown

485 squares). Values represent the mean of three independent determinations made $1 \mathrm{~h}$ apart $486 \pm$ SD. 